\title{
Compact broadband suspended silicon photonic directional coupler
}

\author{
Hamed Sattari, ${ }^{1, *}$ (1) Alain YuJi Takabayashi, ${ }^{1}$ Yu Zhang, ${ }^{1}$ Peter Verheyen, ${ }^{2}$ \\ Wim Bogaerts, ${ }^{2,3}$ (i) and Niels Quack ${ }^{1}$ (i) \\ ${ }^{1}$ École Polytechnique Fédérale de Lausanne, 1015 Lausanne, Switzerland \\ ${ }^{2}$ Interuniversity Microelectronics Centre (IMEC), Leuven, Belgium \\ ${ }^{3}$ Ghent University-IMEC, Photonics Research Group, Department of Information Technology, Ghent, Belgium \\ *Corresponding author: hamed.sattari@epfl.ch
}

Received 6 April 2020; accepted 23 April 2020; posted 27 April 2020 (Doc. ID 394470); published 26 May 2020

Directional couplers are extensively used in photonic integrated circuits as basic components for efficient on-chip photonic signal routing. Conventionally, directional couplers are fully encapsulated in the technology's waveguide cladding material. In this Letter, we demonstrate a compact broadband directional coupler, fully suspended in air and exhibiting efficient power coupling in the cross state. The coupler is designed and built based on IMEC's iSiPP50G standard platform, and hydrofluoric (HF) vapor-etchingbased post-processing allows to release the freestanding component. A low insertion loss of $0.5 \mathrm{~dB}$ at $\lambda=1560 \mathrm{~nm}$ and a $1 \mathrm{~dB}$ bandwidth of $35 \mathrm{~nm}$ at $\lambda=1550 \mathrm{~nm}$ have been confirmed experimentally. With a small footprint of $20 \mu \mathrm{m} \times 30 \mu \mathrm{m}$ and high mechanical stability, this directional coupler can serve as a basic building block for large-scale silicon photonic microelectromechanical systems (MEMS) circuits. () 2020 Optical Society of America

https://doi.org/10.1364/OL.394470

Provided under the terms of the OSA Open Access Publishing Agreement

Today, standardized silicon photonic platforms are commercially available, offering wafer scale production, including an access to well-developed process design kits with a steadily increasing number of mature library components $[1,2]$. The emerging needs in the field of telecommunication and data center applications are spurring developments toward large-scale integration of photonic components on a single chip, which requires specifically low-power and compact building blocks [3]. Large-scale integration of some of the prominent silicon photonics components, such as thermal and plasma dispersion effect modulators, is challenging as they still suffer from either high power consumption or large footprint. Recently realized plasmonic-based modulators have shown great promise for miniaturization; however, they still present limitations in terms of scaling to large-scale photonic circuits $[4,5]$. For instance, recently demonstrated plasmonic modulators [5] still show a relatively high insertion loss of $\sim 2.5 \mathrm{~dB}$. In addition, the port extinction remains quite limited with $\sim 10 \mathrm{~dB}$, and integration in standard silicon photonics platforms remains elusive. Recent developments in the photonic microelectromechanical systems (MEMS) design and fabrication promise more compact and more power-efficient components. Due to the strong mechanical-optical interaction, shorter propagation lengths are required, and compact devices can be conceived. Additionally, photonic MEMS devices can be made latchable, which enables low-power programmable photonic circuits [6]. Compact silicon photonic MEMS directional couplers have been demonstrated before [7]. Furthermore, large-scale switch arrays [8-10], digital phase shifters [11], tunable ring resonator filters [12], and tunable grating couplers $[13,14]$ have all been realized by integrating MEMS with optical waveguides in silicon photonic platforms. Recently realized high-port-count switch matrices in a two-layer silicon photonics platform [8-10] demonstrate the potential of MEMS for large-scale integration. However, such a two-layer platform is not readily compatible with standard silicon photonic technology offerings, and it has limited possibility to include mature silicon photonics components, such as carrier-depletion-based phase shifters and high-speed detectors next to the MEMS components. To pave the way for integration of the suspended structures and MEMS into standard silicon photonics, we enhance IMEC's iSiPP50G standard silicon photonics platform [15] with the MEMS technology and augment the current library with additional photonic MEMS components [16].

One of the most critical components for such a MEMSenabled silicon photonics platform is a suspended directional coupler, which can be used for power distribution, coupling ratio tuning and switching, and sensing, among others. A compact design allows for large-scale integration in a smaller chip area, and concurrently, a more compact directional coupler is robust against mechanical failures, such as stress-related out-ofplane bending, collapse, and stiction. in addition, a compact freestanding directional coupler design exhibits a reduced mass, which allows for fast photonic MEMS components when integrated with an actuator. Finally, a broadband photonic design is required to support operation over a wide spectral range, and at the same time increase tolerance to dimensional uncertainties due to fabrication, thus allowing scaling to large circuits. We here present the detailed design, simulation, and experimental results of a suspended directional coupler for IMEC's iSiPP50G platform [17,18]. The design is optimized for low-loss full light power transfer to the drop port in the 


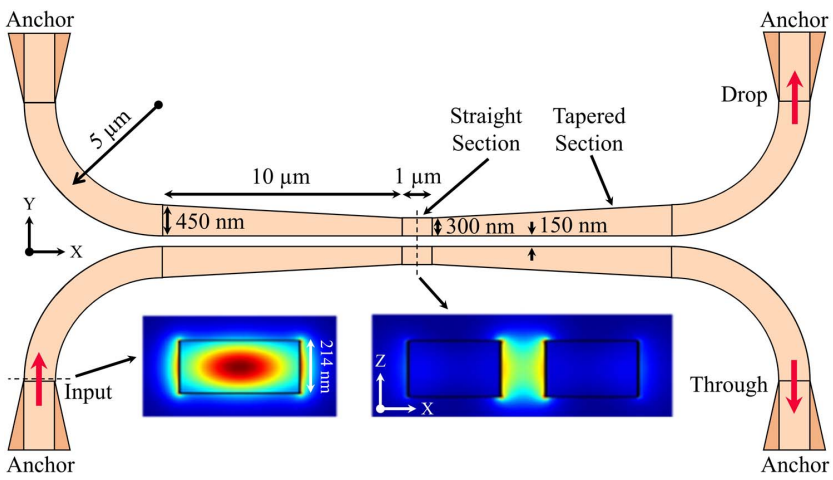

Fig. 1. Schematic of the suspended directional coupler. The electric field profiles in the input and in the middle part of the coupler are depicted in the inset. Dimensions are not to scale.

telecommunication C-band for TE polarization, and it occupies only $20 \mu \mathrm{m} \times 30 \mu \mathrm{m}$. The sketch of the coupler with the related dimensions is presented in Fig. 1.

Here, our motivation is to introduce a design with a short beating length and low coupling loss, which could integrate in a compact large-scale MEMS-enabled silicon photonic circuits. Considering the present silicon photonics platform specifications, and based on the coupled mode theory [19], a straight suspended directional coupler with an air gap of $150 \mathrm{~nm}$ requires an $\sim 30 \mu \mathrm{m}$ beating length. Our design, as an important distinctive feature, for further shrinking the footprint by retaining a low-loss coupling exhibits a tapered waveguide and a short straight section in the middle. The coupler consists of two symmetric arms, each of which has two tapered sections that are connected by a straight section. The suspended waveguide height is considered to be the nominal $214 \mathrm{~nm}$ of the iSiPP50G platform. We choose the gap between the arms to be $150 \mathrm{~nm}$, which is the minimum feature size in the standard technology process and helps the most efficient light coupling. The waveguide width in the tapered sections of the arms reduces from $450 \mathrm{~nm}$ to $300 \mathrm{~nm}$ along $10 \mu \mathrm{m}$. The coupler at the wider ends terminates with 5 - $\mu$ m-radius bent waveguides that connect the coupler to the optical transitions with shallow-etched tapered-rib-waveguide profiles, which at the same time are the mechanical anchors for the suspended coupler. With this kind of anchoring of the suspended directional coupler, we minimize the risk of out-of-plane bending of the arms due to a possible residual stress in the device layer. The optical transition anchors follow a careful design to safely let the light commute between the air-clad region (MEMS openings) and the oxide-clad back end of line (BEOL) stack [18]. Tapering the waveguides allows for increasing the supermodes index contrast along the coupler while the light propagates toward the central section, which leads to a shorter beating length of $\sim 10 \mu \mathrm{m}$ in our design. To achieve a smooth mode transfer between the waveguide sections with a minimum loss, we have included a 1- $\mu$ m-long, 300-nmwide straight waveguide section between the tapered sections. Furthermore, the tapered sections help broaden the coupling bandwidth. Our design results in a lower insertion loss and higher port extinction in comparison with the former silicon photonic suspended directional couplers [7,9].

In a MEMS-enabled platform, the waveguide spacing can conveniently be varied by suitable MEMS actuation mechanisms [6]. Two straightforward actuation mechanism concepts are shown in Fig. 2(a). We have simulated the coupling behavior of this component for various lateral and vertical gap sizes to evaluate the optical power transmission to the drop and through ports. The three-dimensional (3D) finite difference time domain (FDTD) simulation results at a wavelength of $\lambda=1550 \mathrm{~nm}$ for in-plane, and out-of-plane actuation schemes are shown in Figs. 2(b) and 2(c), respectively. The optical power profile for a fixed lateral gap of $150 \mathrm{~nm}$ and zero vertical displacement of the arms can be seen in Fig. 2(d) and shows highly efficient power transfer to the drop port. The simulations indicate high sensitivity of the coupling performance with respect to the waveguide spacing, which is a desirable property for photonic MEMS applications. The simulation results also show that a $0 \%-100 \%$ power coupling tunability is possible with both in-plane (pull and push) and out-of-plane actuation regimes. For the in-plane pull regime, the lateral gap is increased from 150 to $215 \mathrm{~nm}$ or from 215 to $330 \mathrm{~nm}$ to fully couple the power from one port to the other. In the in-plane push regime, the full-power-switching action is accessible by decreasing the gap from 150 to $100 \mathrm{~nm}$. The slope in the power-coupling profile is steeper for the smaller gaps because the coupling between the two arms is stronger in this region. For the out-of-plane actuation regime, a $0 \%-100 \%$ power-coupling tunability can be obtained in either of two vertical displacement spans, from 0 to $300 \mathrm{~nm}$ or from 300 to $510 \mathrm{~nm}$. It is worth noting that for the passive state of the component, light couples from the input port to the drop port after one cycle of beating, as shown in Fig. 2(d). This purposefully devised property enables an additional tunability window in power coupling, tuning to either of the ports under actuation. In case of any fabrication errors, such as variation in the gap and waveguide sizes or waveguides misalignment, the waveguides spacing can still be tuned in all the pull, push, and out-of-plane regimes to have a full power transmission to the drop port. Because of the high sensitivity of the coupling behavior to changes in the lateral gap and the vertical displacement, only a small displacement is required for the switching action. This small displacement can, for example, be applied by a compact comb drive actuator or an extended electrode area in one of the arms, for in-plane movement or out-of-plane displacement, respectively.

To verify the mechanical robustness of the design against stress-related issues, we performed a 3D finite element method (FEM) simulation. The suspended directional coupler is anchored to a silicon frame surrounding the MEMS opening box via a set of shallow-etched optical-waveguide transitions. To set up a precise model, we define a set of fixed boundary conditions on the silicon frame, spaced at $2 \mu \mathrm{m}$ from edge of the frame, which represents the final isotropic etch front of the $2-\mu \mathrm{m}$-thick BOX. Although the crystalline silicon device layer is not expected to retain a high level of intrinsic stress, we have studied behavior of the component when it goes under extreme levels of residual stress. Typically, the residual stress bends the structure out-of-plane or shrinks/elongates them depending on type of the residual stress. In Fig. 3, we have plotted three displacement field components for $300 \mathrm{MPa}$ of compressive and tensile stresses. Figure 3 depicts a negligible out-of-plane displacement for the coupler arms, even when a high value of the intrinsic stress is applied in the material properties of the model. This amount of out-of-plane displacement does not substantially affect the coupler's optical performance [see Fig. 2(c)], because the displacement is much smaller than the 


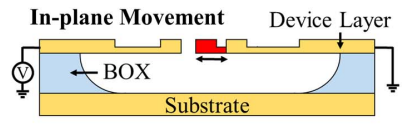

(a)

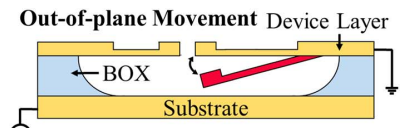

Actuated State
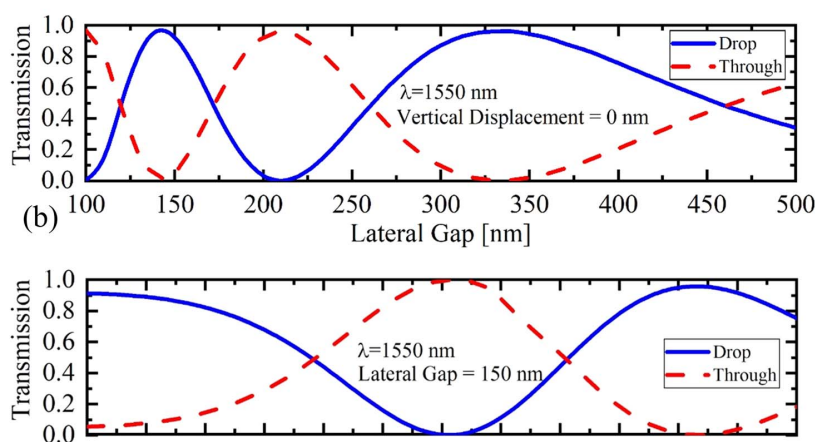

(c) $\quad \begin{array}{lllllllllllll}50 & 100 & 150 & 200 & 250 & 300 & 350 & 400 & 450 & 500 & 550 & 600\end{array}$ Vertical Displacement [nm]

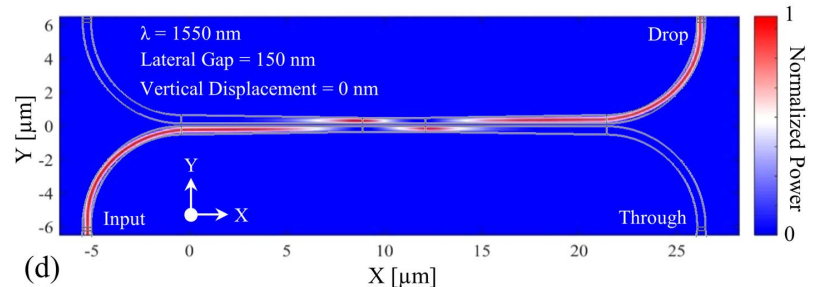

Fig. 2. (a) Possible actuation mechanisms for a suspended directional coupler. The simulated transmission in the drop and through ports of the coupler versus (b) the lateral air gap between the coupled waveguides and (c) the vertical displacement of one of the coupler's arms at $\lambda=1550 \mathrm{~nm}$. (d) The electrical power profile in the coupler for a lateral gap of $150 \mathrm{~nm}$ and a vertical displacement of $0 \mathrm{~nm}$ at $\lambda=1550 \mathrm{~nm}$.

waveguide height $(214 \mathrm{~nm})$, and the coupler arms remain in the coupling state. As expected, using the bent waveguides for connecting the coupler to the anchors prevents the structure from out-of-plane buckling. However, the Y component (u), which affects the lateral gap between the waveguides, becomes considerable when the stress value is high. Such a gap change can be compensated in an active device by applying a small offset in the actuation voltage. For a realistic case we expect $\sim 17 \mathrm{MPa}$ of compressive stress in the device layer, which causes a coupler length expansion of $1.5 \mathrm{~nm}$, a lateral gap shrinkage of $5 \mathrm{~nm}$, and no out-of-plane bending. These variations can lead to a slight shift in the power-coupling spectrum. Furthermore, from an eigenfrequency simulation, we extracted a fundamental frequency of $0.668 \mathrm{MHz}$ for the suspended directional coupler, which relates its stiffness and promises for microsecond-range actuation speed possibilities.

The test device is fabricated in a simplified version of IMEC's iSiPP50G standard silicon photonics technology platform, without several active technology modules such, as detectors, modulators, and metal routings, but with layer stacks of equivalent composition and thickness, including a passive finishing stack comparable to the full-process BEOL. Realizing any suspended structure in the device layer requires removal of the BEOL stack on top of the device layer. In the opening window area, the device is exposed to air but still rests atop a $2 \mu \mathrm{m}$ BOX. After receiving the wafer with the BEOL openings from IMEC, we carry out post-processing steps at the Center

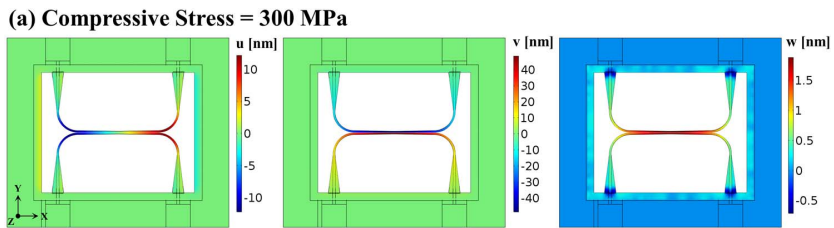

(b) Tensile Stress $=300 \mathrm{MPa}$

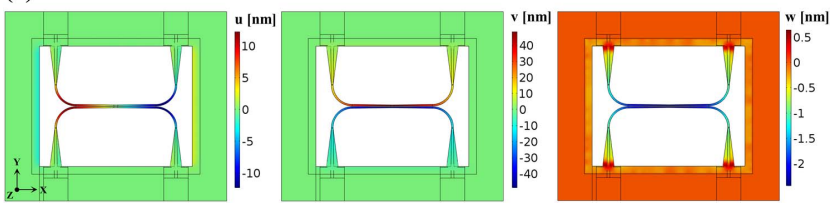

Fig. 3. Displacement field components for the suspended directional coupler under $300 \mathrm{MPa}$ of (a) compressive stress and (b) tensile stress. The $u, v$, and $w$ correspond to the displacement field components in the $X, Y$, and $Z$ directions, respectively.

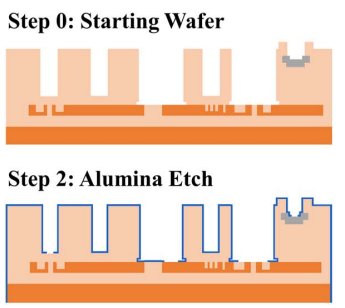

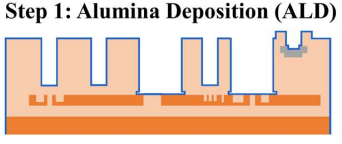

Step 3: VHF Release

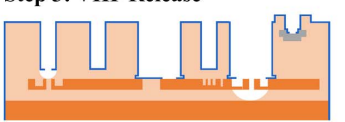

Oxide
Fig. 4. Fabrication process flow for the post-processing on the wafer to release the suspended structures [18]. VHF, vapor hydrofluoric acid.

of MicroNanoTechnology at EPFL to selectively etch the BOX in regions where we have MEMS structures that should be suspended. The post-processing fabrication flow is shown in Fig. 4. First, we deposit $50 \mathrm{~nm}$ of alumina $\left(\mathrm{Al}_{2} \mathrm{O}_{3}\right)$ with an atomic layer deposition technique using a BENQ TSF200 tool to protect the BEOL from the final hydrofluoric (HF) vapor-etching step. Next, we etch the alumina layer in the opening windows using the STS Multiplex inductive coupled plasma (ICP) etcher to give the $\mathrm{HF}$ vapor access to the $\mathrm{BOX}$ beneath the structures. Finally, we release the MEMS structures using vapor phase HF isotropic etching with the SPTS uEtch machine to remove the $2 \mu \mathrm{m}$ BOX layer below the silicon on insulator (SOI) device layer. Scanning electron microscope (SEM) micrographs of the BEOL with the openings and the suspended directional coupler after the HF vapor etching are presented in Figs. 5(a), 5(b), respectively. Upon an inspection, it can be observed that the component is released and the arms retain excellent alignment, which corroborates the claim that design's compactness and stiff anchors lend it mechanical robustness. According to
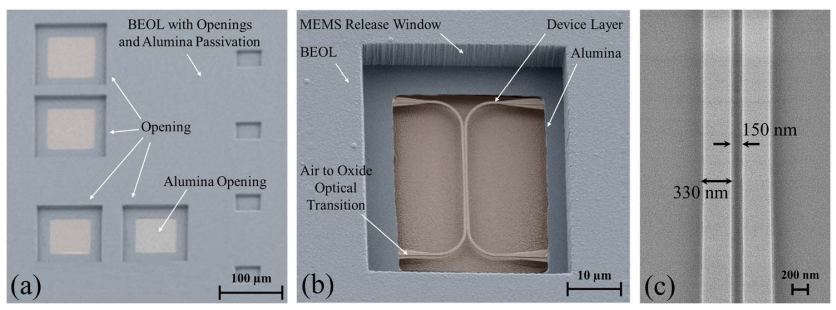

Fig. 5. (a) BEOL with openings and alumina passivation. (b) Released suspended directional coupler. (c) Closer view of the waveguides in the middle part of the coupler just before the release step. 

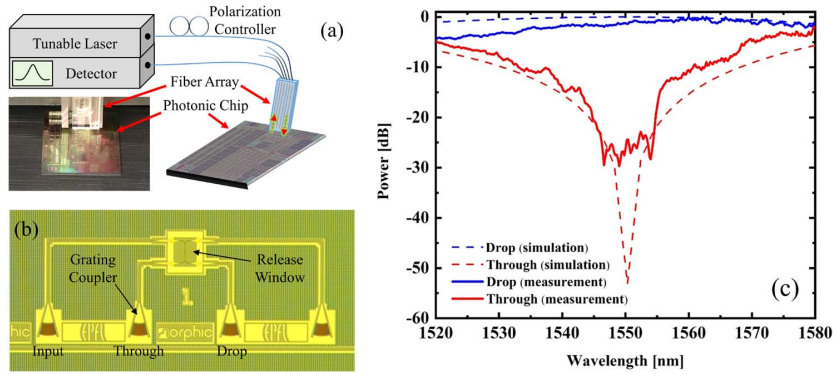

Fig. 6. (a) Schematic of the characterization setup. (b) Image of the photonic chip under test. (c) Optical microscope image of the chip area. (d) Transmitted power spectrum to the drop and the through ports of the coupler overlaid with the FDTD simulation results.

Fig. 5(b), the suspended coupler arms are well-anchored with the optical transition structures, and the alumina passivation of the BEOL has prevented the HF vapor from interacting with the BEOL layers. Consequently, there is no byproduct material, which could introduce scattering loss, residing on the coupler or waveguides. For further investigation on the precision of the fabrication for defining the minimum feature and gap sizes, we took a chip from the same wafer and measured the width of the waveguide sections on a directional coupler before the postprocessing steps. From the SEM image in Fig. 5(c), we measured a $330 \mathrm{~nm}$ width for the straight waveguide section, which was supposed to be $300 \mathrm{~nm}$ as designed. This corresponds to the width bias between design and fabrication, as documented in IMEC's iSiPP50G platform documentation. Still, the gap between the waveguides is measured at $150 \mathrm{~nm}$, as defined in the design.

A schematic of the characterization setup is shown in Fig. 6(a). Light from a tunable laser (Agilent 8164A) is coupled into the photonic chip, using an array of single-mode fibers and the integrated vertical grating couplers. An image of the photonic chip with the fiber array is also shown in Fig. 6(a). An optical microscope image of the chip area is presented in Fig. 6(b). The measurement results for the drop and through ports of the device with the overlaid 3D FDTD simulated power spectrum are shown in Fig. 6(c), which exhibit a good agreement between the simulation and the measurement. The coupler exhibits an insertion loss of $0.5 \mathrm{~dB}$ at $\lambda=1560 \mathrm{~nm}$ and a $1 \mathrm{~dB}$ bandwidth of $35 \mathrm{~nm}$ at $\lambda=1550 \mathrm{~nm}$, which fully covers the telecommunication C-band. We also measure a large port extinction ratio of $\sim 25 \mathrm{~dB}$ at $\lambda=1550 \mathrm{~nm}$. We note that the measured extinction ratio at $\lambda=1550 \mathrm{~nm}$ is smaller than predicted by simulation, which can be attributed to sidewall roughness of the waveguides, effectively modulating the supermode index and the coupling length of the central wavelength so that a portion of the light leaks to the through port, and consequently, the extinction ratio drops. Due to the larger index contrast between the silicon waveguide and the air cladding compared to an oxide cladding, the scattering loss arising from the sidewall roughness [20] contributes to the insertion loss of the component, yet due to the compactness of the device, the excess loss is kept minimal, as confirmed by the experiment.

In conclusion, in this Letter, we present a compact, suspended directional coupler for a MEMS-enabled standard silicon photonics platform. The coupler is composed of a set of tapered and narrow, straight sections, which facilitate low-loss efficient power coupling in a short propagation length. The coupler is fabricated in a simplified flow of IMEC's iSiPP50G silicon photonics platform. The freestanding directional coupler is safely released using an HF vapor post-processing. The suspended directional coupler guides the light to the drop port with a $0.5 \mathrm{~dB}$ insertion loss at $\lambda=1560 \mathrm{~nm}$ and has a $1 \mathrm{~dB}$ bandwidth of $35 \mathrm{~nm}$ at $\lambda=1550 \mathrm{~nm}$. This compact coupler with an $\sim 20 \mu \mathrm{m} \times 30 \mu \mathrm{m}$ footprint can serve as a key library component for a MEMS-enabled silicon photonics platform, addressing the emerging need for large-scale integrated photonic circuits.

Funding. Horizon 2020 Framework Programme (780283); Hasler Stiftung (17008); Schweizerischer Nationalfonds zur Förderung der Wissenschaftlichen Forschung (183717).

Disclosures. The authors declare no conflict of interest.

\section{REFERENCES}

1. A. Rahim, T. Spuesens, R. Baets, and W. Bogaerts, Proc. IEEE 106, $2313(2018)$.

2. A. E. Lim, J. Song, Q. Fang, C. Li, X. Tu, N. Duan, K. K. Chen, R. P. Tern, and T. Liow, IEEE J. Sel. Top. Quantum Electron. 20, 405 (2014).

3. Q. Cheng, S. Rumley, M. Bahadori, and K. Bergman, Opt. Express 26, 16022 (2018).

4. L. Thylén and L. Wosinski, Photon. Res. 2, 75 (2014).

5. C. Haffner, D. Chelladurai, Y. Fedoryshyn, A. Josten, B. Baeuerle, W. Heni, T. Watanabe, T. Cui, B. Cheng, S. Saha, D. L. Elder, L. R. Dalton, A. Boltasseva, V. M. Shalaev, N. Kinsey, and J. Leuthold, Nature 556, 483 (2018).

6. C. Errando-Herranz, A. Y. Takabayashi, P. Edinger, H. Sattari, K. B. Gylfason, and N. Quack, IEEE J. Sel. Top. Quantum Electron. 26, 8200916 (2020).

7. Y. Akihama and K. Hane, Sci. Appl. 1, e16 (2012).

8. T. J. Seok, N. Quack, S. Han, R. S. Muller, and M. C. Wu, Optica 3, 64 (2016).

9. S. Han, T. J. Seok, N. Quack, B.-W. Yoo, and M. C. Wu, Optica 2, 370 (2015).

10. T. J. Seok, K. Kwon, J. Henriksson, J. Luo, and M. C. Wu, Optica 6 , 490 (2019).

11. J. Henriksson, T. J. Seok, J. Luo, K. Kwon, N. Quack, and M. C. Wu, in International Conference on Optical MEMS and Nanophotonics (OMN) (2018), pp. 1-2.

12. C. Errando-Herranz, F. Niklaus, G. Stemme, and K. B. Gylfason, Opt. Lett. 40, 3556 (2015).

13. C. Errando-Herranz, M. Colangelo, S. Ahmed, J. Björk, and K. B. Gylfason, in IEEE 30th International Conference on Micro Electro Mechanical Systems (MEMS) (2017), pp. 293-296.

14. W. Yu, S. Gao, Y. Lin, M. He, L. Liu, J. Xu, Y. Luo, and X. Cai, IEEE Photon. Technol. Lett. 31, 161 (2019).

15. P. Absil, K. Croes, A. Lesniewska, P. De Heyn, Y. Ban, B. Snyder, J. De Coster, F. Fodor, V. Simons, S. Balakrishnan, and G. Lepage, in IEEE International Electron Devices Meeting (IEDM) (IEEE, 2017) p. 34.

16. W. Bogaerts, H. Sattari, P. Edinger, A. Y. Takabayashi, I. Zand, X. Wang, A. Ribeiro, M. Jezzini, C. Errando-Herranz, G. Talli, K. Saurav, M. G. Porcel, P. Verheyen, B. Abasahl, F. Niklaus, N. Quack, K. B. Gylfason, P. O'Brien, and U. Khan, Proc. SPIE 11285, 1128503 (2020).

17. H. Sattari, Y. Zhang, Y. Takabayashi, and N. Quack, in International Conference on Optical MEMS and Nanophotonics (OMN) (IEEE, 2019), pp. 214-215.

18. N. Quack, H. Sattari, A. Y. Takabayashi, Y. Zhang, P. Verheyen, W. Bogaerts, P. Edinger, C. Errando-Herranz, and K. B. Gylfason, IEEE J. Quantum Electron. 56(1), 8400210 (2019).

19. E. A. J. Marcatili, Bell Syst. Tech. J. 48, 2071 (1969).

20. D. Melati, F. Morichetti, and A. Melloni, J. Opt. (Paris) 16, 055502 (2014). 